\title{
Panax notoginseng saponins alleviate osteoporosis and joint destruction in rabbits with antigen-induced arthritis
}

\author{
CHEN CHAO WEI ${ }^{1}$, LI FEI YUE ${ }^{2}$, FAN TIAN YOU $^{1}$ and CHE TAO ${ }^{1}$ \\ ${ }^{1}$ Department of Orthopaedics and Traumatology, Shanghai Municipal Hospital of Traditional \\ Chinese Medicine, Shanghai University of TCM, Shanghai 200071; ${ }^{2}$ Department of Traumatology, \\ Rui Jin Hospital Shanghai Jiao Tong University School of Medicine, Shanghai 200025, P.R. China
}

Received October 22, 2020; Accepted May 17, 2021

DOI: $10.3892 /$ etm.2021.10737

\begin{abstract}
Although a number of anti-rheumatic drugs and biologics may be used to alleviate the symptoms of rheumatoid arthritis (RA), these compounds have been associated with bone loss and joint destruction; thus, alternative treatment approaches are required. In the present study, various plant extracts were evaluated for their capacity to inhibit joint destruction, and Panax notoginseng saponins (PNS), obtained from the Traditional Chinese Medicine Panax notoginseng, was identified as such a compound. Therefore, a rabbit antigen-induced arthritis (AIA) model was generated by immunization with ovalbumin in Freund's complete adjuvant, followed by treatment with PNS for 3 months. The morphology of the quadriceps femoris muscle, cartilage chondrocytes and skeletal elements was histologically observed by transmission electron microscopy (TEM), as well as micro-computed tomography. The results revealed that PNS significantly reduced the histopathological alterations associated with arthritic muscular atrophy and inflammation. In addition, TEM demonstrated that PNS protected chondrocytes from RA-associated damage. Furthermore, the bone density and microarchitecture in rabbits treated with PNS were markedly improved compared with those of the model group. Collectively, these data indicated that treatment with PNS may relieve osteoporosis and prevent joint and bone destruction in AIA.
\end{abstract}

\section{Introduction}

Rheumatoid arthritis (RA) is a chronic immune-mediated inflammatory disease characterized by deformity of the joints and functional disability (1). Limb swelling, pain, stiffness and bone erosion at the joint have been reported to primarily result

Correspondence to: Dr Che Tao, Department of Orthopaedics and Traumatology, Shanghai Municipal Hospital of Traditional Chinese Medicine, Shanghai University of TCM, 274 Zhijiang Middle Road, Shanghai 200071, P.R. China

E-mail: chetao7117@163.com

Key words: antigen-induced arthritis, Panax notoginseng saponins, rheumatoid arthritis, osteoporosis, joint destruction from inflammatory cytokine release and propagation in the synovial joint (2). Furthermore, it is now accepted that osteoporosis in RA is associated with an imbalance between bone formation and absorption, leading to a decrease in bone mineral density (BMD) and subsequent deterioration of the trabecular microstructure (3). RA-associated chronic synovitis is largely due to the expression of proinflammatory cytokines, such as IL-17, TNF- $\alpha$, IL-1 $\beta$ and macrophage colony-stimulating factor (M-CSF), which independently stimulate osteoclast differentiation, thereby significantly increasing bone resorption and formation (4).

Panax notoginseng saponins (PNS) is the primary active compound obtained from the Traditional Chinese Medicinal plant Panax notoginseng, which has been used to treat musculoskeletal injuries in China for hundreds of years (5). PNS was previously shown to possess various pharmacological properties, including its beneficial effects on cardiomyocytes and brain cells (6). Furthermore, PNS may attenuate atherogenesis by promoting blood circulation and it may also exert immunoregulatory effects according to a previous study (7). PNS has also been shown to exert a considerable clinical effect in the relief of RA symptoms (8). Furthermore, Cai et al (9) reported that PNS had noticeably improved the clinical manifestations of joint pain, tenderness and swelling associated with RA at the 2-year follow-up. PNS prevents the progression of RA, which may be associated with a decrease in the numbers of Th17 and Th1 cells, and inhibits the production of INF- $\gamma$ and IL-17 (10). PNS has also been reported to stimulate bone formation in excess of resorption, and to inhibit osteoclast activity in vitro (11). Jang et al (12) reported that the PNS-induced regulation of MMPs and osteoclastogenesis is responsible, in part, for its bone-protective effects. Therefore, it was hypothesized that PNS may be used to treat RA-associated joint destruction and osteoporosis. The present study was undertaken to investigate the mechanism through which PNS relieves joint destruction, in the hope of providing further experimental evidence to support the clinical use of PNS in the treatment of patients with RA.

\section{Materials and methods}

Animals and treatments. PNS powder was purchased from Beijing Qihuang Pharmaceutical Co., Ltd. Indomethacin was used as the positive control treatment; each tablet contained 
$25 \mathrm{mg}$ indomethacin, and was purchased from Mundipharma International Ltd. A total of 37 adult female New Zealand rabbits, aged 6 months and weighing 2.5-3 kg, were purchased from the Beijing Experimental Animal Center. According to previous research (13), based on the total success rate of model establishment, estimation of sample size through power calculations using a two-sided t-test indicated that $\sim 6$ rabbits would be required for each group. Therefore, 37 rabbits in total were included in this trial. All rabbits were allowed access to water and food ad libitum and were housed at a temperature of $23^{\circ} \mathrm{C}$ and humidity of $45 \%$, with a 12 -h light/dark cycle. Body weight, food intake and animal health were examined daily.

The antigen-induced arthritis (AIA) model was generated as previously described (13). Briefly, all rabbits were allowed to acclimate for 1 week, and 30 of the 37 rabbits were then subcutaneously immunized with $500 \mu \mathrm{g}$ ovalbumin in complete Freund's adjuvant emulsion (Sigma-Aldrich; Merck KGaA). Injections were administered once a week for 2 weeks, and the AIA rabbits were then boosted with $100 \mu \mathrm{g}$ ovalbumin via and intra-articular injection into the knee joints, once a week for the following 4 weeks.

After 6 weeks of treatment, all 30 AIA rabbits were scored according to the symptoms presented in Table I (14); 21 rabbits with clinical symptom scores $>5$ were randomized into three groups. The first arthritic rabbit group did not receive any treatment (AIA, n=7). The second arthritic rabbit group was treated with PNS by means of intragastric administration (gavage) at a dose of $75 \mathrm{mg} / \mathrm{kg}$ body weight per day (PNS, $\mathrm{n}=7$ ); PNS ( $75 \mathrm{mg} / \mathrm{kg}$ body weight per day) was diluted in distilled water and each rabbit was administered $20 \mathrm{ml}$ by gavage. The third arthritic rabbit group received indomethacin by gavage at a dose of $10 \mathrm{mg} / \mathrm{kg}$ body weight per day (Control, $\mathrm{n}=7$ ); indomethacin powder (10 mg/kg body weight) was diluted with normal saline and shaken to generate a $20-\mathrm{ml}$ suspension for gavage. The rationale for selecting a dose of $10 \mathrm{mg} / \mathrm{kg}$ was that it equates to 9 times the clinical dose for a $70-\mathrm{kg}$ adult. Rabbits in the normal group $(n=7)$ did not receive treatment of any type, and had free access to distilled water and food. At 3 months post-treatment, the rabbits were sacrificed with a lethal dose of sodium pentobarbital (100 mg/kg, intravenous), and death was confirmed by the absence of a heartbeat and visible breathing. The knee joint and first lumbar vertebra were then collected for further experimentation. All procedures were authorized by the Animal Care Committee of Shanghai University of Traditional Chinese Medicine (Shanghai, China). All animal experimental protocols were conducted in accordance with the Institutional Animal Care and Use Committee of Shin Nippon Biomedical Laboratories, Ltd. and the National Institutes of Health Guide for Care and Use of Laboratory Animals (15).

Morphological analysis of skeletal muscle. Histopathological analysis of selected muscles was conducted. The quadriceps femoris muscle of the knee joint was dissected from the left side, washed in $\mathrm{PBS}$, fixed at $4^{\circ} \mathrm{C}$ overnight in $4 \%$ polyformaldehyde, and then dehydrated in a graded ethanol series. Tissue sections were embedded in paraffin and cut into $4-\mu \mathrm{m}$ sections, and then stained at $25^{\circ} \mathrm{C}$ with $1 \%$ hematoxylin and eosin (H\&E) for histopathological evaluation using an BX54 optical microscope (Olympus Corporation; magnification, $\mathrm{x} 300)$.
Table I. Criteria for arthritis activity score.

\begin{tabular}{lc}
\hline Arthritis symptoms & Score \\
\hline No joint swelling & 0 \\
Redness or swelling of one joint & 1 \\
Redness and swelling of more than one joints & 2 \\
Ankle and tarsal-metatarsal joint involvement & 3 \\
Entire leg and paw redness or swelling & 4
\end{tabular}

Total arthritis symptom scores was calculated by adding the four individual scores. Scores $>5$ were considered to indicate successful antigen-induced arthritis model establishment.

Processing for transmission electron microscopy (TEM). Articular cartilage pieces from the left distal femur were prepared for TEM according to the procedure described by Chen et al (16). In order to remove organic material, the samples were treated with $10 \%$ EDTA for 3 weeks at a temperature of $30^{\circ} \mathrm{C}$ followed by increasing concentrations of alcohol $(75-100 \%)$, slowly embedded at $37^{\circ} \mathrm{C}$ in $100 \%$ Epon 812 resin for $48 \mathrm{~h}$, and then cut into $50-\mathrm{nm}$ sections. Tissue samples were double-stained at $37^{\circ} \mathrm{C}$ with $2 \%$ uranyl and $1 \%$ phosphotungstic acid for TEM analysis.

Skeletal microstructure analysis. As previously described (17), the right distal femur and the first lumbar vertebra were prepared for micro-computed tomography (micro-CT) examination using a Locus SP scanner (GE Healthcare). The microstructural parameters of the trabeculae, such as trabecular thickness, trabecular bone volume fraction (BV/TV), trabecular spacing (Tb.Sp), trabecular number (Tb.N) and BMD, were evaluated.

Statistical analysis. Continuous variables are presented as the mean \pm SD. Mean differences between the control and experimental groups were compared by one-way ANOVA followed by Tukey's post hoc test. All statistical analyses were performed using SPSS 25.0 (IBM Corp.), and two-sided $\mathrm{P}<0.05$ was considered to indicate a statistically significant difference.

\section{Results}

PNS reduces arthritic muscular atrophy and inflammation. The morphological characteristics of rabbit skeletal muscle fibers were observed following H\&E staining. Skeletal muscle from the normal group (in longitudinal sections) exhibited a compact structure with no gaps between the fibers, deeply stained nuclei in a linear arrangement around intact muscle fiber cells, and few inflammatory cells. The AIA group exhibited various degrees of inflammatory cell infiltration into the empty spaces between fibers, and the skeletal muscle fibers displayed increased atrophy compared with the other groups. However, narrower intercellular spaces and less prominent fiber atrophy were observed in the PNS-treated muscle fibers. In addition, cross sections of the skeletal muscle fibers in the PNS-treated groups revealed a considerable decrease in inflammatory cell numbers. Moreover, the number of 

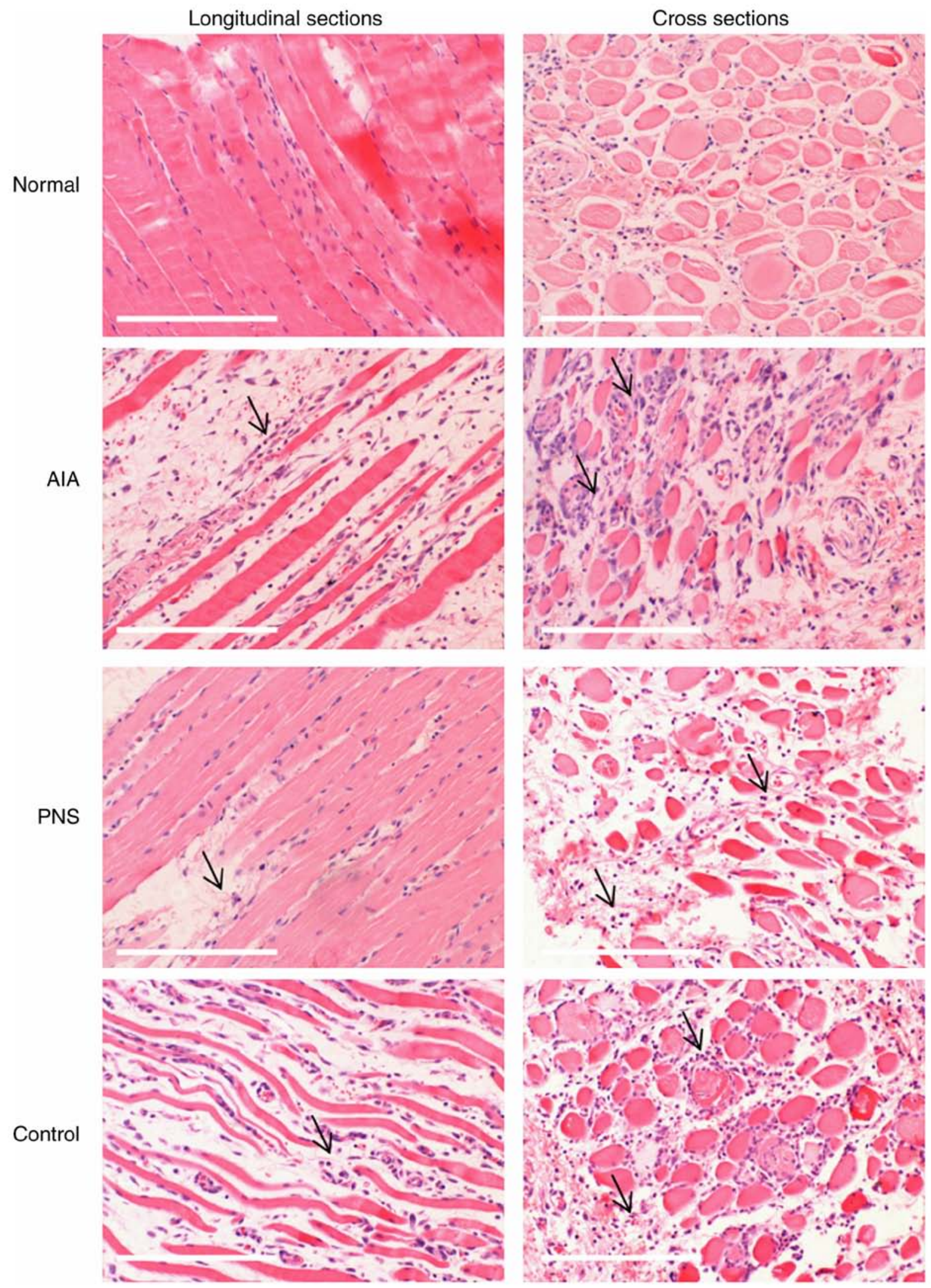

Figure 1. Histopathological characteristics of the quadriceps femoris muscle were analyzed by hematoxylin and eosin staining. Degeneration of the necrotic quadriceps femoris muscle fibers with inflammatory cell infiltration (arrows) were observed in the model and treatment groups. Original magnification, $\mathrm{x} 300$; scale bar, $200 \mu \mathrm{m}$. AIA, antigen-induced arthritis; PNS, Panax notoginseng saponins.

inflammatory cells in the indomethacin-treated control group was lower compared with that in the AIA group (Fig. 1).
PNS inhibits articular chondrocyte apoptosis. TEM images of articular cartilage and the extracellular matrix (ECM) are 

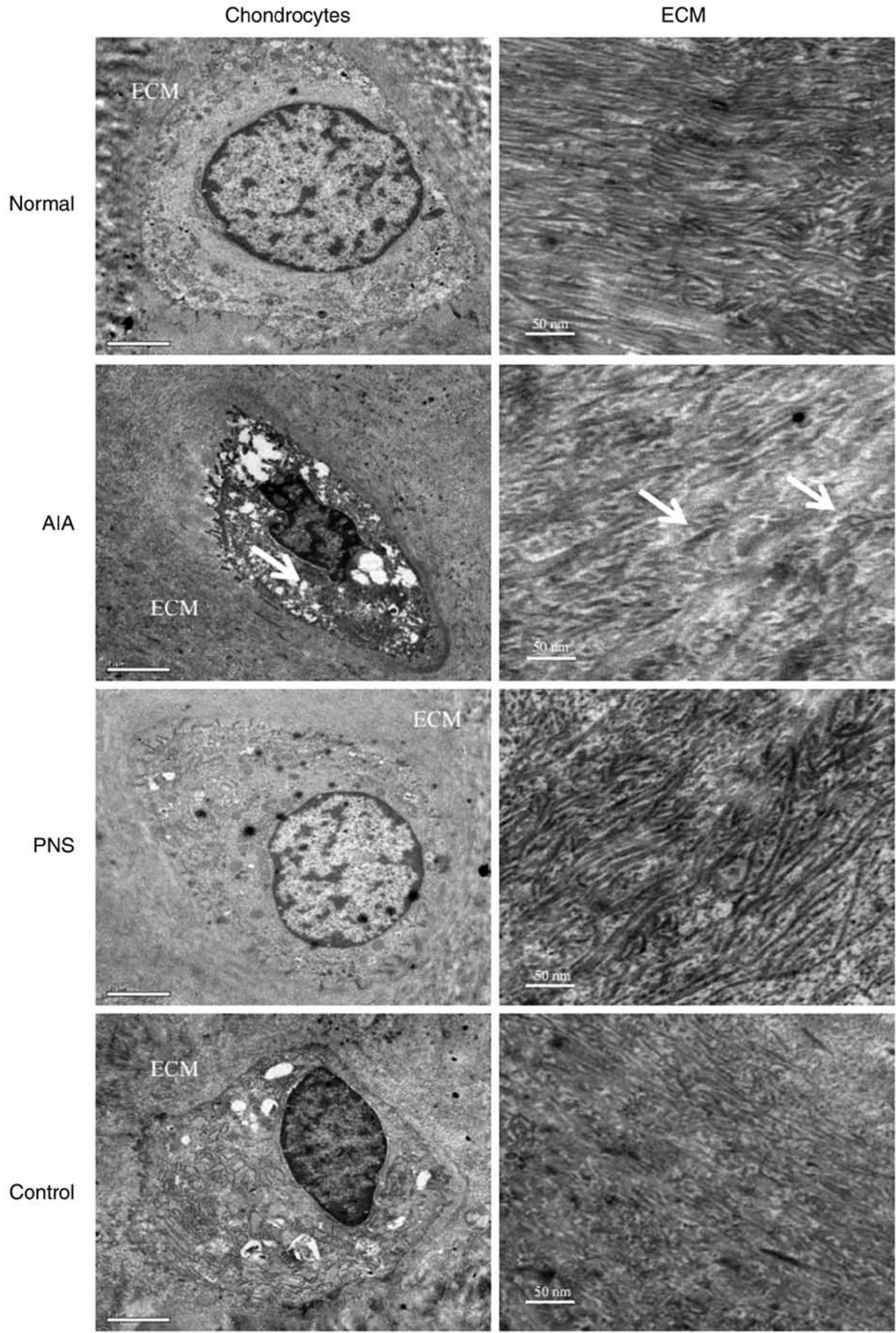

Figure 2. TEM images of chondrocytes and ECM of the articular cartilage. The number of intracellular organelles, such as mitochondria and Golgi bodies, was markedly reduced in the AIA chondrocytes, and short fibril forms and numerous abnormal collagen heterofibrils (arrows) were observed in the ECM. Chondrocytes: Original magnification, $\mathrm{x} 10,000$; scale bar, $2 \mu \mathrm{m}$. ECM: Original magnification, $\mathrm{x} 12,000$; scale bar, $50 \mathrm{~nm}$. TEM, transmission electron microscopy; ECM, extracellular matrix; AIA, antigen-induced arthritis; PNS, Panax notoginseng saponins. 


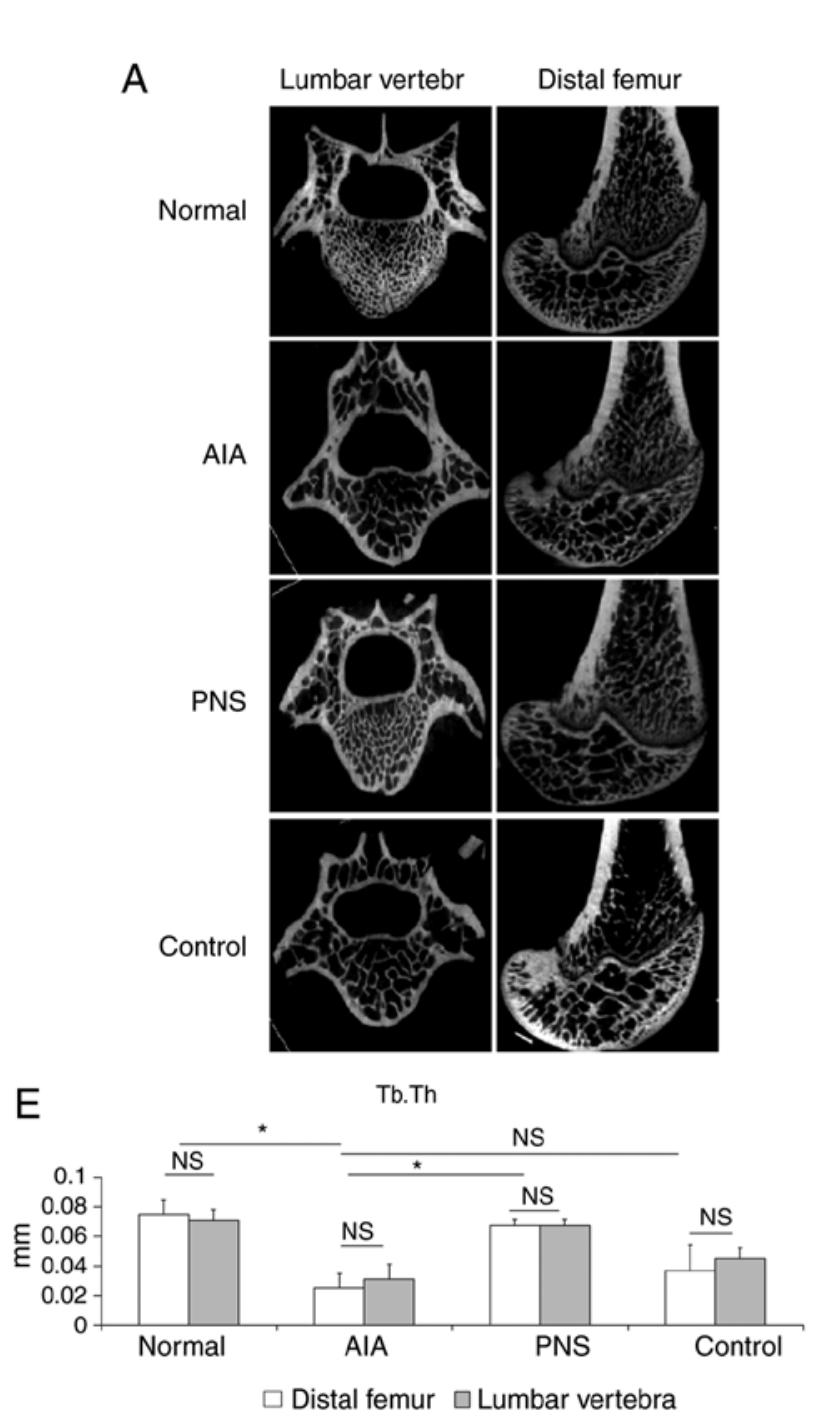

B

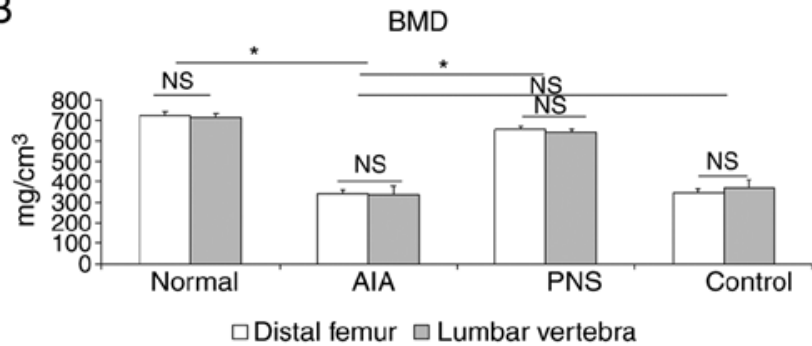

C

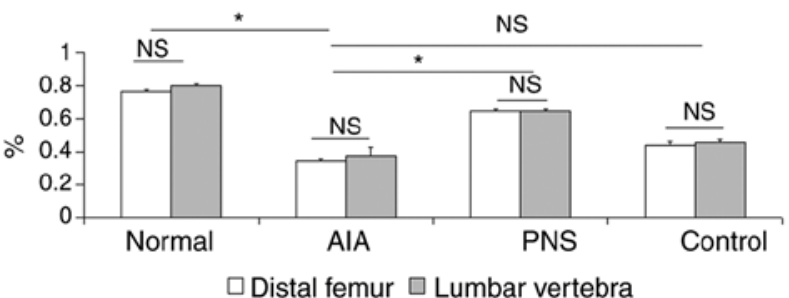

D

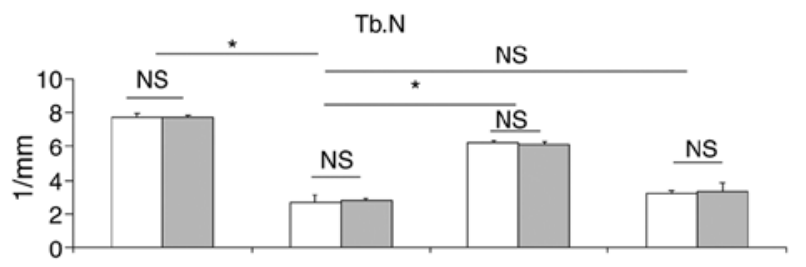

$\square$ Distal femur $\quad$ Lumbar vertebra

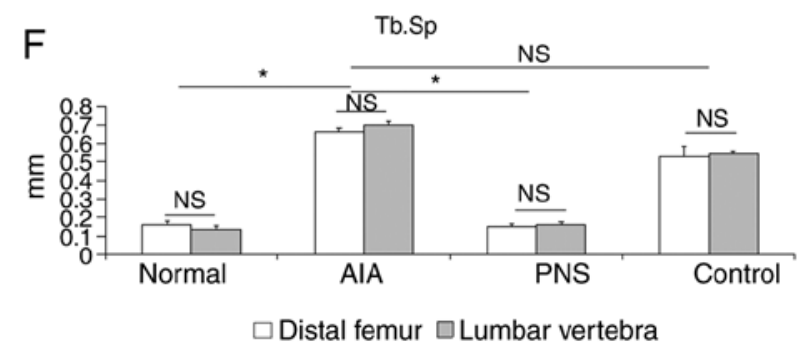

Figure 3. Micro-CT analysis of the BMD value, bone structure and quality of the femoral condyle and the first lumbar vertebra. (A) Micro-CT images of the femoral condyle and the first lumbar vertebra of rats from the different groups. (B) Comparison of BMD values among the groups. Compared with model rabbits, (C) BV/TV, (D) Tb.N (D) and (E) Tb.Th were significantly increased, whereas (F) Tb.Sp was significantly decreased in PNS-treated rabbits. The results are presented as the mean \pm SD. ${ }^{*} \mathrm{P}<0.05$. NS, not significant; Micro-CT, micro-computed tomography; AIA, antigen-induced arthritis; PNS, Panax notoginseng saponins; BMD, bone mineral density; BV/TV, trabecular bone volume fraction; Tb.N, trabecular number; Tb.Sp, trabecular spacing; Tb.Th, trabecular thickness.

displayed in Fig. 2. Examination of the TEM images revealed that the typical chondrocyte exhibited heterochromatic regions within the cell nucleus. The endoplasmic reticulum was expansive and prominent, the Golgi bodies were highly developed, and numerous mitochondria were visible. Altered chondrocyte ultrastructure was apparent in the AIA model; the cells had irregular hyperchromatic nuclei, and the cell membrane possessed short filopodia. Moreover, the intracellular organelles, such as mitochondria and Golgi bodies, were markedly reduced, whereas short fibril forms and numerous abnormal collagen heterofibrils were observed in the ECM. However, treatment with PNS decreased chondrocyte apoptosis. In addition, the cytoplasm was shown to contain an abundance of active organelles, and the cell membrane possessed longer filopodia in the rabbits treated with PNS compared with the control group. Furthermore, a highly complex ECM with a visible underlying collagen fibril meshwork, as well as fibers, was observed in the PNS-treated group compared with the control group.
PNS inhibits lumbar vertebral and articular bone destruction. Micro-CT revealed that AIA-induced osteoporosis was evident in the femoral condyle and lumbar spine (Fig. 3A). The BMD value in both the distal femoral and the lumbar vertebra was significantly lower in the model rabbits compared with the normal rabbits $(\mathrm{P}<0.05)$. By contrast, the BMD value of the PNS-treated group was higher compared with that of the model rabbits $(\mathrm{P}<0.05$; Fig. 3B). Furthermore, AIA significantly decreased trabecular BV/TV, Tb.Th and Tb.N compared with the normal group; by contrast, $\mathrm{Tb} . \mathrm{Sp}$ was increased as a result of AIA-induced osteoporosis $(\mathrm{P}<0.05)$. Moreover, as regards the microarchitecture of the trabecular bone in the PNS-treated group, the BV/TV, Tb.Th and Tb.N were markedly increased $(\mathrm{P}<0.05)$, and Tb.Sp was significantly decreased $(\mathrm{P}<0.05)$. PNS significantly inhibited microstructural deterioration of the trabeculae. However, indomethacin did not significantly affect the BMD and microarchitecture of the trabecular bone when compared with the AIA group ( $\mathrm{P}>0.05)$. Furthermore, the degree of apoptosis was compared between the lumbar 
vertebra and knee joint in the same group, and the data indicated that differences in the BMD value and microarchitecture of the trabecular bone did not achieve statistical significance (P>0.05; Fig. 3C-F).

\section{Discussion}

RA is a progressive inflammatory disorder that involves not only bone and joint destruction, but also muscle wasting. RA results in osteoporosis, impaired bone and joint function (18); thus, preventing osteoporosis and joint damage is crucial for RA treatment (19). AIA is a well-developed experimental arthritis model that is widely used in the study of RA $(20,21)$. Unlike the ovariectomy-induced osteoporosis model (22), joint swelling and a pluricellular infiltrate are observed in AIA, which may progress to a chronic condition characterized by synovial inflammation and matrix degradation. Specifically, the severity of synovitis in animal models may be modified by altering the number or dosage of the intra-articular injections (13). In the present study, the AIA experimental model was generated using injection of ovalbumin in complete Freund's adjuvant emulsion, resulting in severe synovial lesions and osteoporosis. Shen et al (22) reported that PNS at a dose of $300 \mathrm{mg} / \mathrm{kg} /$ day significantly prevented the ovariectomy-induced reduction of bone mass in rats. Therefore, in the present study, the $75 \mathrm{mg} / \mathrm{kg}$ dose of PNS in rabbits was calculated by converting the rat dosage, and the results demonstrated that PNS exerted a positive effect on bone loss and cartilage erosion in a rabbit model of inflammatory arthritis.

The pathological characteristics of RA include joint destruction and muscle wasting. Multiple factors promote muscle atrophy in RA, such as joint pain, swelling and local inflammation. It is also known that RA-associated muscle atrophy may be mediated by pro-inflammatory factors, such as IL-17, TNF- $\alpha$ and inducible nitric oxide synthase (iNOS) (23). Yamada et al (24) reported that muscles from AIA rats exhibited increased levels of TNF- $\alpha$ and high-mobility group box 1, as well as increased NO and superoxide production, leading to the formation of actin aggregates and decreased actomyosin ATPase activity in skeletal muscles, ultimately resulting in intrinsic contractile dysfunction of skeletal muscles. Previous evidence indicated that PNS treatment improved joint tenderness and swelling in collagen-induced arthritic rats by reducing the expression of pro-inflammatory factors, including MMPs, iNOS and TNF- $\alpha$ (25). In the present study, AIA was shown to cause inflammatory dysfunction in skeletal muscles. Degeneration and necrosis of the muscle fibers, reduced muscle bundle width and irregularities in muscle fiber size were observed in AIA animals. The muscle fibers in the PNS-treated groups were less atrophic, and the number of inflammatory cells was markedly lower. These findings indicate the potential therapeutic value of PNS for muscle wasting in patients with rheumatic conditions or other inflammatory diseases. Indomethacin, which is a non-steroidal anti-inflammatory drug commonly used in patients with RA, was used as the control drug in the present study. It was previously demonstrated that indomethacin significantly inhibited pro-inflammatory cytokine expression in the synovial tissues of AIA rats (26). In the present study, the number of inflammatory cells in the indomethacin-treated control group was lower compared with that in the AIA group.
Articular cartilage comprises ECM and chondrocytes. Collagen II is the main structural protein component of the ECM (27), and chondrocyte injury plays a crucial role in joint destruction in RA (28). As previously reported (29), chondrocytes from the cartilage of the normal group possessed centrally located nuclei, the staining had a finely granular appearance, and the chondrocytes were encompassed by the ECM, while, in damaged cartilage, the chondrocytes were encompassed by a reduced amount of cartilaginous matrix (30). In the present study, TEM images revealed chondrocyte apoptosis in the AIA model, resulting in failure to form an adequate quantity of cytoplasm and cellular organelles, such as endoplasmic reticulum, mitochondria and Golgi bodies. However, chondrocytes were adequately preserved in rabbits treated with PNS, and the collagen fibrils in the ECM were denser and thicker compared with those in the model rabbits.

Micro-CT was previously used to determine whether PNS could maintain bone quality, and the BMD values of the lumbar vertebrae and knee joints in the PNS group were higher compared with those in the AIA model group (31). Systemic osteoporosis in RA has a complex multifactorial etiology, in which an autoimmune inflammatory response plays a significant role. Bone loss in RA, as evidenced by the release of osteoclast-activating cytokines (for example, IL-17 and M-CSF) by synovial cells, directly enhanced the expression of tumor necrosis factor ligand superfamily member 11 (TNFSF11) (4). Various bioactive compounds derived from foods or plants, such as icariin and theaflavins, have been shown to prevent bone loss, decrease osteoclastic bone resorption, and stimulate osteoblast activity in AIA models $(17,26)$. Our previous study indicated that the levels of TNFSF11 and Osteoprotegerin expression were directly associated with bone formation and resorption in AIA rabbits (16). Further analysis of the trabecular microarchitecture revealed that the BV/TV, Tb.Th and Tb.N were also significantly increased, whereas trabecular separation was markedly decreased following PNS treatment, suggesting that PNS may exert a therapeutic effect on osteoporosis. These results are consistent with previous findings according to which PNS prevented ovariectomy-related osteoporosis in rats, and that these beneficial effects may be the result of stimulating bone formation and decreasing resorption (31). In conclusion, the results of the present study indicated that PNS may relieve osteoporosis and prevent joint bone destruction in rabbits with AIA.

\section{Acknowledgements}

Not applicable.

\section{Funding}

The present study was supported by the Shanghai Science and Technology Commission (grant no. 19401935400), the construction project of Li Fei Yue's National Famous TCM (grant no. MLZJGZS-2017001), the Construction Project of Li Fei Yue's Shanghai Famous TCM Research Studio (grant no. SHGZS-2017010) the National Natural Science Foundation of China (grant no. 81302987) and the National Training Program for Innovative Talents of Traditional Chinese Medicine. 


\section{Availability of data and materials}

The datasets used and/or analyzed during the current study are available from the corresponding author on reasonable request.

\section{Authors' contributions}

LFY and CCW designed the study. CCW drafted the manuscript. FTY performed the experiments. CCW and CT verified and statistically analyzed the data, and proofread the manuscript . All the authors have read and approved the final manuscript. CCW and CT confirm the authenticity of all the raw data.

\section{Ethics approval and consent to participate}

The present study was approved by the Medical Ethics Committee of the Animal Care Committee of Shanghai University of Traditional Chinese Medicine (approval no. 201601217117).

\section{Patient consent for publication}

Not applicable.

\section{Competing interests}

The authors declare that they have no competing interests.

\section{References}

1. Croia C, Bursi R, Sutera D, Petrelli F, Alunno A and Puxeddu I: One year in review 2019: Pathogenesis of rheumatoid arthritis. Clin Exp Rheumatol 37: 347-357, 2019.

2. Gao W, McGarry T, Orr C, McCormick J, Veale DJ and Fearon U: Tofacitinib regulates synovial inflammation in psoriatic arthritis, inhibiting STAT activation and induction of negative feedback inhibitors. Ann Rheum Dis 75: 311-315, 2016.

3. Gaudio A, Pennisi P, Bratengeier C, Torrisi V, Lindner B, Mangiafico RA, Pulvirenti I, Hawa G, Tringali G and Fiore CE: Increased sclerostin serum levels associated with bone formation and resorption markers in patients with immobilization-induced bone loss. J Clin Endocrinol Metab 95: 2248-2253, 2010.

4. Kwan Tat S, Padrines M, Théoleyre S, Heymann D and Fortun Y: IL-6, RANKL, TNF-alpha/IL-1: Interrelations in bone resorption pathophysiology. Cytokine Growth Factor Rev 15: 49-60, 2004.

5. Pumpa KL, Fallon KE, Bensoussan A and Papalia S: The effects of Panax notoginseng on delayed onset muscle soreness and muscle damage in well-trained males: A double blind randomised controlled trial. Complement Ther Med 21: 131-140, 2013.

6. Zhou N, Tang Y, Keep RF, Ma X and Xiang J: Antioxidative effects of Panax notoginseng saponins in brain cells. Phytomedicine 21: 1189-1195, 2014.

7. Liu Y, Zhang HG, Jia Y and Li XH: Panax notoginseng saponins attenuate atherogenesis accelerated by zymosan in rabbits. Biol Pharm Bull 33: 1324-1330, 2010.

8. Li XY: Immunomodulating Chinese herbal medicines. Mem Inst Oswaldo Cruz 86 (Suppl 2): S159-S164, 1991.

9. Cai H, Zhang QY, Zhao ZM and Yao RB: Two years follow-up of Panax notoginseng saponins combined with DMARDs on treating rheumatoid arthritis. J Med Postgrad 26: 505-507, 2013 (In Chinese)

10. Wei JR, Wen X, Bible PW, Li Z, Nussenblatt RB and Wei L: Panax notoginseng saponin controls IL-17 expression in helper T cells. J Ocul Pharmacol Ther 33: 285-289, 2017.

11. Li XD, Chang B, Chen B, Liu ZY, Liu DX, Wang JS, Hou GQ, Huang DY and Du SX: Panax notoginseng saponins potentiate osteogenesis of bone marrow stromal cells by modulating gap junction intercellular communication activities. Cell Physiol Biochem 26: 1081-1092, 2010.
12. Jang YJ, Kim ME and Ko SY: n-Butanol extracts of Panax notoginseng suppress LPS-induced MMP-2 expression in periodontal ligament fibroblasts and inhibit osteoclastogenesis by suppressing MAPK in LPS-activated RAW264.7 cells. Arch Oral Biol 56: 1319-1327, 2011.

13. Largo R, Roman-Blas JA, Moreno-Rubio J, Sánchez-Pernaute O, Martínez-Calatrava MJ, Castañeda S and Herrero-Beaumont G: Chondroitin sulfate improves synovitis in rabbits with chronic antigen-induced arthritis. Osteoarthritis Cartilage 18 (Suppl 1): S17-S23, 2010.

14. Ochaion A, Bar-Yehuda S, Cohn S, Del Valle L, Perez-Liz G, Madi L, Barer F, Farbstein M, Fishman-Furman S, Reitblat T, et al: Methotrexate enhances the anti-inflammatory effect of CF101 via up-regulation of the A3 adenosine receptor expression. Arthritis Res Ther 8: R169, 2006.

15. Derrell CJ, Gebhart GF, Gonder JC, Keeling ME and Kohn DF: Special report: The 1996 guide for the care and use of laboratory animals. ILAR J 38: 41-48, 1997.

16. Chen CW, Fan TY, Li YM, Sun J and Chen YQ: Total glucosides of paeony prevents juxta-articular bone loss in experimental arthritis. BMC Complement Altern Med 13: 186, 2013.

17. Wei CC, Ping DQ, You FT, Qiang CY and Tao C: Icariin prevents cartilage and bone degradation in experimental models of arthritis. Mediators Inflamm 2016: 9529630, 2016.

18. Jang EJ, Lee YK, Choi HJ, Ha YC, Jang S, Shin CS and Cho NH: Osteoporotic fracture risk assessment using bone mineral density in Korean: A community-based cohort study. J Bone Metab 23: 34-39, 2016.

19. Maruotti N, Corrado A and Cantatore FP: Osteoporosis and rheumatic diseases. Reumatismo 66: 125-135, 2014.

20. Lei Z, Feng G, Xu N, Wei Q, Liu J,Bian T and Zou T: Early extremity MRI findings and pathological synovial changes in antigen-induced arthritis rabbit model. J Magn Reson Imaging 39: 1366-1373, 2014.

21. Lewthwaite J, Blake SM, Hardingham TE, Warden PJ and Henderson B: The effect of recombinant human interleukin 1 receptor antagonist on the induction phase of antigen induced arthritis in the rabbit. J Rheumatol 21: 467-472, 1994.

22. Shen Y, Li YQ, Li SP, Ma L, Ding LJ and Ji H: Alleviation of ovariectomy-induced osteoporosis in rats by Panax notoginseng saponins. J Nat Med 64: 336-345, 2010.

23. Horai N, Nagaoka T, Higuchi I, Kasai H, Yoshioka T, Umekita Y, Fukuzaki K, Nagata R, Miyata A and Abeyama K: Muscle wasting associated with pathologic change is a risk factor for the exacerbation of joint swelling in collagen-induced arthritis in cynomolgus monkeys. BMC Musculoskelet Disord 14: 205, 2013.

24. Yamada T, Abe M, Lee J, Tatebayashi D, Himori K, Kanzaki K, Wada M, Bruton JD, Westerblad H and Lanner JT: Muscle dysfunction associated with adjuvant-induced arthritis is prevented by antioxidant treatment. Skelet Muscle 5: 20, 2015.

25. Chang SH, Choi Y, Park JA, Jung DS, Shin J, Yang JH, Ko SY, Kim SW and Kim JK: Anti-inflammatory effects of BT-201, an n-butanol extract of Panax notoginseng, observed in vitro and in a collagen-induced arthritis model. Clin Nutr 26: 785-791, 2007.

26. Ramadan G, El-Beih NM, Talaat RM and Abd El-Ghffar EA: Anti-inflammatory activity of green versus black tea aqueous extract in a rat model of human rheumatoid arthritis. Int J Rheum Dis 20: 203-213, 2017.

27. Raya JG: Techniques and applications of in vivo diffusion imaging of articular cartilage. J Magn Reson Imaging 41: 1487-1504, 2015.

28. Huh YH, Lee G, Lee KB, Koh JT, Chun JS and Ryu JH: HIF-2 $\alpha$-induced chemokines stimulate motility of fibroblast-like synoviocytes and chondrocytes into the cartilage-pannus interface in experimental rheumatoid arthritis mouse models. Arthritis Res Ther 17: 302, 2015.

29. Toh ML, Bonnefoy JY, Accart N, Cochin S, Pohle S, Haegel H, De Meyer M, Zemmour C, Preville X, Guillen C, et al: Bone- and cartilage-protective effects of a monoclonal antibody against colony-stimulating factor 1 receptor in experimental arthritis. Arthritis Rheumatol 66: 2989-3000, 2014.

30. Bustamante MF, Garcia-Carbonell R, Whisenant KD and Guma M: Fibroblast-like synoviocyte metabolism in the pathogenesis of rheumatoid arthritis. Arthritis Res Ther 19: 110, 2017.

31. Fan JZ, Wang Y, Meng Y, Li GW, Chang SX, Nian H and Liang YJ: Panax notoginseng saponins mitigate ovariectomy-induced bone loss and inhibit marrow adiposity in rats. Menopause 22: 1343-1350, 2015

This work is licensed under a Creative Commons Attribution-NonCommercial-NoDerivatives 4.0 International (CC BY-NC-ND 4.0) License. 